\title{
FATORES PROGNÓSTICOS PARA VENTILAÇÃO MECÂNICA EM LACTENTES COM DOENÇA RESPIRATÓRIA AGUDA BAIXA
}

\author{
Adriana Gut lopes Riccetto*, José Dirceu Ribeiro, Marcos tadeu Nolasco da Silva, Emílio Carlos Elias Baracat
}

Trabalho realizado na Universidade Estadual de Campinas - UNICAMP, SP

*Correspondência:

Rua Washington Luís, 752, apto 112, Centro

Americana, SP

CEP: $13465-520$

Fone: (19) 3462-1514

a.riccetto@uol.com.br

\begin{abstract}
RESUMO
OBjEtIvo. Infecções respiratórias agudas são a causa mais comum de internação hospitalar em pediatria. Uma proporção de pacientes internados necessita de ventilação pulmonar mecânica invasiva (VPMI). Este estudo objetivou avaliar fatores prognósticos para VPMI em lactentes hospitalizados por infecção respiratória aguda baixa.

Métodos. Estudo de coorte prospectivo, realizado de abril a setembro de 2004, em dois hospitais universitários da região de Campinas, São Paulo, Brasil. Foram avaliados 152 lactentes internados, registrando-se dados epidemiológicos e clínicos na internação e no seguimento. Analisaram-se dois grupos, de acordo com a necessidade de VPMI, comparando-se os fatores prognósticos. A associação entre fatores de risco e desfecho estudado foi analisada por risco relativo (RR) e intervalos de confiança (IC 95\%).
\end{abstract}

Resultados. Vinte e um pacientes $(13,81 \%)$ necessitaram de VPMI. Fatores significativamente associados à VPMI na admissão foram: idade < 3 meses ( $R R=2,35$, IC 95\%: I,06-5,22), aleitamento materno < I mês (RR=3, I I, IC 95\%: I,35$7,35)$ e cianose (RR=7,55, IC 95\%:5,0 I- II,36). Observou-se risco aumentado no grupo de VPMI para internação > 10 dias ( $R R=13,69$, IC 95\%:4,92-38,09), oxigenioterapia > 10 dias ( $R R=13,57$, IC 95\%:5,41-34,03), uso de antibióticos $(\mathrm{RR}=3,03, \mathrm{IC} 95 \%: 1,34-6,89)$ e reinternações (RR=5,23, IC 95\%:2, I2-I2,91).

ConcLusä́o. As associações entre a necessidade de VPMI e baixa idade, tempo curto de aleitamento materno e cianose à admissão demonstram as reduzidas reservas fisiológicas no lactente jovem com infecção respiratória baixa. Caracterizam este grupo de pacientes a necessidade de suporte hospitalar prolongado e intensivo e maior taxa de reinternações.

UnITERMOS: Infecção respiratória aguda. Ventilação mecânica. Terapia intensiva pediátrica. Crianças.

\section{INTRODUÇÃO}

As infecções agudas do trato respiratório são as doenças mais comuns em crianças e adultos; ocorrem em todas as partes do mundo; porém, nos países em desenvolvimento, levam a maior mortalidade, principalmente na faixa etária menor de 12 meses $(0,7 / 100.000$ na França versus 25I/I00.000 na Guatemala - década de 1980), constituindo-se objeto prioritário de atenção de políticas públicas de saúdel,2. No Brasil, as doenças respiratórias são responsáveis por mais de $40 \%$ das consultas médicas e mais de $30 \%$ das admissões hospitalares; nestas, cerca de $10 \%$ dos pacientes evoluem para óbito². Na última década, a mortalidade por infecções respiratórias agudas vem diminuindo, assim como a taxa de mortalidade por esta causa em crianças menores de 5 anos (50 para 10/1000/ano).

Do total das infecções agudas envolvendo o trato respiratório, calcula-se que cerca de 10\% acometem o trato respiratório inferior. Aproximadamente uma em cada quatro crianças menores de 12 meses é avaliada por pediatra, a cada ano, por infecções das vias aéreas inferiores ${ }^{4}$. A apresentação e a evolução destes pacientes são variáveis, de acordo com o agente causal $\left.\right|^{5}$ e a doença desencadeada; os casos mais graves estão freqüentemente associados às bronquiolites $e$ pneumonias com insuficiência respiratória!. Considerando somente as infecções causadas por vírus sincicial respiratório (VSR), ocorrem cerca de 123.000 hospitalizações/ano de crianças menores de 12 meses nos Estados Unidos da América, com gastos anuais estimados de 300 a 400 milhões de dólares ${ }^{6}$.

As infecções respiratórias baixas (especialmente aquelas causadas pelo VSR e parainfluenza) podem levar a episódios recorrentes de sibilância por toda a infância ${ }^{7}$, particularmente mais freqüentes no primeiro ano de vida. Em estudo com 499 crianças, demonstrou-se que o risco relativo (RR) de dois ou mais episódios de sibilos no primeiro ano de vida foi maior para as infecções respiratórias baixas prévias $(R R=2,25)$ do que para tabagismo materno durante a gravidez $(R R=1,83)$, peso baixo ao nascer $(R R=1,28)$ e alergia a baratas no ambiente domiciliar $(\mathrm{RR}=1,63)^{8}$.

Tem-se verificado como fatores de risco para doença respiratória: idade (lactentes jovens e idosos), aglomeração (lares com maior número de irmãos e creches); sexo masculino, desnutrição protéico-calórica, ausência de aleitamento materno e poluição ambiental (tabagismo materno e uso de lenha e gás dentro dos domicílios) $)^{9,10}$. No entanto, não existem evidências consistentes da relação entre condições socioeconômicas desfavoráveis e baixa escolaridade materna e risco para doença respiratória aguda baixa ${ }^{10,11}$. Em relação à evolução clínica desfavorável, os fatores mais fortemente associados a pior prognóstico são as anormalidades anatômicas, metabólicas, imunológicas e genéticas'. 
Nos pacientes internados por doença respiratória aguda baixa, existe risco potencial de evolução para falência respiratória e necessidade de suporte ventilatório. Analisando os fatores de risco para intubação endotraqueal e ventilação pulmonar mecânica invasiva (VPMI), Willson et al., em estudo envolvendo 684 crianças hospitalizadas por bronquiolite ou pneumonia, encontraram I $4 \%$ destas crianças evoluindo para a necessidade de VPMI, sendo este risco muito maior em crianças com doenças prévias (28,7\% para prematuros, $31,7 \%$ para cardiopatas e 37,3\% para crianças portadoras de outras anomalias congênitas) do que em crianças sem doenças anteriores $(9,1 \%)^{12}$.

O objetivo do presente estudo foi avaliar fatores prognósticos para VPMI de lactentes menores de 12 meses hospitalizados por infecções respiratórias agudas baixas e sua evolução clínica.

\section{Métodos}

Estudo observacional, analítico, longitudinal, do tipo coorte prospectivo.

Foram acompanhados lactentes admitidos em enfermaria geral de pediatria, em dias definidos previamente, sendo às quartas e sextasfeiras no Hospital das Clínicas (HC) da Universidade Estadual de Campinas e às segundas e quintas-feiras no Hospital Estadual Sumaré (HES). Os dois hospitais universitários estão localizados na região metropolitana de Campinas, em área abrangendo população com características socioeconômicas semelhante' ${ }^{13}$. Os responsáveis pelos pacientes autorizaram sua participação após leitura do termo de consentimento esclarecido; o projeto foi aprovado pelo Comitê de Ética em Pesquisa da Faculdade de Ciências Médicas da Universidade Estadual de Campinas, sob o número 076/2003, em I5/04/2003.

Foram incluídas no estudo crianças de 0 a 12 meses, hospitalizadas no $\mathrm{HC}$ e no HES no período de I de abril a 30 de setembro de 2004 e que receberam o diagnóstico inicial de doença respiratória aguda baixa, definida pelos sinais clínicos de taquidispnéia, ausculta pulmonar alterada e oximetria de pulso menor que $95 \%$ em ar ambiente. Foram excluídas crianças com doença respiratória com duração maior que sete dias e aquelas com instabilidade hemodinâmica, cardiopatias ou doença pulmonar crônica comprovada, pois tais condições podem cursar com alterações clínicas e radiológicas erroneamente interpretadas como secundárias à infecção respiratória aguda.

Em cada paciente foi aplicado um questionário e feito registro para os dados epidemiológicos, de exame físico à admissão e evolutivos na internação. Os dados epidemiológicos considerados foram: gênero, etnia, idade em meses, peso ao nascer, idade gestacional, tempo de aleitamento materno, presença de doenças concomitantes, episódios anteriores de sibilância, número de tabagistas no domicílio, número de pessoas no domicílio, idade e escolaridade maternas. No exame físico à admissão hospitalar, verificaram-se freqüência cardíaca e respiratória ${ }^{14}$; presença ou não de cianose; oximetria de pulso em ar ambiente; presença ou ausência de retrações, sibilos, estertores e assimetria à ausculta. $\mathrm{Na}$ avaliação durante a hospitalização, verificaram-se tempo de hospitalização, tempo de oxigenioterapia, necessidade de ventilação mecânica, uso de antibióticos e corticoterapia. A indicação de VPMI foi definida pelo médico assistente no momento do atendimento, segundo critérios e protocolos de conduta já estabelecidos em consensos na literatura médica. Não havia conhecimento por parte desta equipe médica dos objetivos do presente trabalho.

Para todos os pacientes foi programada avaliação no $7^{\circ}, 15^{\circ}$ e $30^{\circ}$ dia após a admissão hospitalar, feita por exame físico e entrevista durante o período de hospitalização ou em consultas ambulatoriais programadas. Para aqueles pacientes que não compareceram aos retornos ambulatoriais, foi feito contato por telefone. Para análise dos dados, os pacientes foram divididos em dois grupos: com e sem necessidade de ventilação mecânica.

A análise estatística dos resultados foi realizada pela Câmara de Pesquisa - Setor de Estatística, FCM-UNICAMP; foi feita a análise descritiva por meio de medidas de posição e dispersão para variáveis contínuas e tabelas de freqüência para variáveis categóricas. O cálculo do tamanho amostral baseou-se no número total de internações pediátricas de 0 a 12 meses de idade e da proporção de crianças com doenças respiratórias agudas baixas hospitalizadas no ano anterior. A análise de risco foi realizada pelo cálculo do risco relativo (RR) com intervalo de confiança para 95\% (IC 95\%). Foram utilizados os programas computacionais SpSS for Windows I I. O e SAS System for Windows 8.2.

\section{Resultados}

Foram avaliados I 52 pacientes, 59 (38,8\%) do sexo feminino e 93 $(61,2 \%)$ do sexo masculino. Noventa e sete pacientes $(63,8 \%)$ eram caucasóides e 55 (36,2\%) negróides. Vinte e um pacientes (13,8\%) evoluíram para VPMI, com média de idade menor em relação aos não entubados (3,57 meses versus 4,6 meses). Comparando-se os grupos de idade menor e maior que 3 meses, observou-se um risco maior para VPMI entre os menores de 3 meses (RR: 2,35; IC 95\%; I,06-5,22).

Nos dois grupos houve predominância do sexo masculino. A maior parte das crianças apresentava peso ao nascer $\geq 3000 \mathrm{~g}$ e idade gestacional > 37 semanas, não se observando diferenças entre os dois grupos avaliados. Quanto ao tempo de aleitamento materno, os lactentes não amamentados ou amamentados por período menor de um mês apresentaram risco maior de evolução para VPMI (RR: 3, I ; IC 95\%: I,35-7,35).

A maioria dos pacientes era previamente hígida $(73,68 \%)$ e nunca havia apresentado sibilos anteriormente $(71,71 \%)$, sem diferença entre os dois grupos. Ausência de diferença significante entre os grupos também foi verificada em relação a tabagismo dos cuidadores, idade materna, aglomeração doméstica (média de 4,85 pessoas/domićlio) e escolaridade materna.

Nos parâmetros clínicos à admissão, não ocorreram diferenças com relação à frequiência respiratória, presença de taquicardia, saturação de $\mathrm{O}$, $<90 \%$ e ausculta do tórax. Houve risco maior para evolução para VPMI nas crianças com cianose (RR: 7,55, IC 95\%:5,0 I - I I,36) à admissão.

$\mathrm{Na}$ evolução, observou-se maior tempo de hospitalização (RR: I3,69; IC 95\%: 4,92-38,09) e de oxigenioterapia no grupo em VPMI, assim como maior uso de antibióticos (RR: 3,03: IC 95\%: I,34$6,86)$ e maior número de reinternações no período de 30 dias (RR: 5,23; IC 95\%: 2, I2-I2,9I). Não ocorreu diferença para o uso de corticoterapia (Tabela I). 


\begin{tabular}{|c|c|c|c|}
\hline Variável & $\begin{array}{c}\text { Grupo } \\
\text { ventilaçẫo mecânica } \\
\text { (presença/ausência) }\end{array}$ & $\begin{array}{c}\text { Gruposem } \\
\text { ventilaçấo mecânica } \\
\text { (presença/ausência) }\end{array}$ & $\begin{array}{l}\text { Risco relativo } \\
\text { (IC 95\%)* }\end{array}$ \\
\hline Idade $<3$ meses & $9 / 12$ & $43 / 88$ & $2,35(1,06-5,22)$ \\
\hline Aleitamento materno $<$ I mês & $14 / 7$ & $45 / 86$ & $3,15(1,35-7,35)$ \\
\hline Clanose & $1 / 20$ & $0 /|3|$ & $7,55(5,01-11,36)$ \\
\hline Internação $>10$ dias & $17 / 4$ & $19 / 112$ & $13,69(4,92-38,09)$ \\
\hline Oxigenioterapia $>10$ dias & $16 / 5$ & $13 / 118$ & $\mid 3,57(5,4 \mid-34,03)$ \\
\hline Uso de antimicrobianos & $13 / 8$ & $40 / 91$ & $3,03(1,34-6,86)$ \\
\hline Reinternação & $2 / 19$ & $1 / 130$ & $5,23(2,|2-12,9|)$ \\
\hline Sexo masculino & $14 / 7$ & $79 / 52$ & $1,26(0,54-2,96)$ \\
\hline Etnia caucasóide & $16 / 5$ & $81 / 50$ & ।,81 (0,70-4,68) \\
\hline Peso de nascimento < $2500 \mathrm{~g}$ & $4 / 16$ & $17 / 114$ & $1,55(0,57-4,18)$ \\
\hline $\mid$ dade gestacional $<35$ semanas ao nascer & $3 / 17$ & $9 / 122$ & $2,04(0,69-5,99)$ \\
\hline Doenças prévias & $7 / 14$ & $33 / 98$ & $1,40(0,61-3,22)$ \\
\hline Sibilo anterior & $3 / 18$ & $40 / 91$ & $0,42(0,13-1,36)$ \\
\hline Tabagismofamillar & $4 / 17$ & $54 / 77$ & $0,38(0,12-1,08)$ \\
\hline Mais de 5 moradores no domićlilio & $13 / 8$ & $67 / 64$ & $1,46(0,64-3,32)$ \\
\hline Idade materna $<20$ anos & $3 / 17$ & $38 / 93$ & $0,47(0,15-1,32)$ \\
\hline Escolaridade materna $<5$ anos & $3 / 17$ & $32 / 97$ & $0,57(0,18-1,85)$ \\
\hline Taquicardia & $12 / 9$ & $46 / 85$ & $2,16(0,97-4,81)$ \\
\hline Saturação de $\mathrm{O}_{2}<90 \%$ & $8 / 13$ & $25 / 106$ & $2,22(1,00-4,90)$ \\
\hline Corticoterapia & $5 / 16$ & $17 / 114$ & $1,85(0,75-4,53)$ \\
\hline
\end{tabular}

* Associações significativas destacadas em negrito

\section{Dıscussão}

A avaliação das 152 crianças deste estudo mostrou risco significativo para ventilação mecânica nos pacientes menores de 3 meses. As diferenças anatômicas e fisiológicas do sistema respiratório nesta faixa etária, particularmente quanto ao número de alvéolos, fibras musculares lisas na parede bronquial, complacência da parede torácica e características próprias do diafragma, podem explicar a maior tendência à insuficiência respiratória e conseqüente necessidade de ventilação mecânica neste grupo de crianças ${ }^{15}$. Estes dados reforçam a preocupação na abordagem clínica e diagnóstica desta faixa etária, já que existe baixa capacidade de compensação e adaptação frente a um agravo infeccioso.

Outro ponto a ser destacado refere-se ao tempo curto (menor de 30 dias) ou ausência de aleitamento materno nas crianças na faixa etária até 3 meses, em que se concentrou a maior parte dos pacientes que evoluíram para VPMI. A importância desta forma de alimentação na prevenção de doenças infecciosas e também de doenças crônicas, como a asma, já está bem determinada na literatura ${ }^{16}$. Estudo realizado no Brasil, com I I 3 crianças com bronquiolite viral aguda, mostrou que o risco de hospitalização foi sete vezes maior para crianças com tempo de aleitamento materno menor que um mês, nos três primeiros meses de vida ${ }^{17}$.
Evidenciou-se também que as crianças de mães com menor tempo de escolaridade não apresentaram maior risco para VPMI. Tal achado é antagônico ao observado em outro estudo brasileiro, em que foram avaliadas 49 I crianças com doença respiratória aguda e verificou-se que naquelas com infecção das vias aéreas inferiores e pior evolução houve associação significativa com menor escolaridade materna ${ }^{18}$. No presente trabalho, o fácil acesso aos serviços de saúde na região, a infra-estrutura hospitalar instalada e o diagnóstico e tratamento precoces podem ter minimizado a influência da educação materna no prognóstico.

$\mathrm{Na}$ avaliação do exame físico à admissão, o grupo de crianças que evoluiu para VPMI não apresentou freqüência cardíaca mais elevada ou saturação transcutânea de oxigênio menor que $90 \%$, parâmetros que refletem um acometimento pulmonar mais acentuado; entretanto, houve maior risco na presença de cianose, sinal clínico de falência respiratória. Em um estudo com 748 crianças hospitalizadas por pneumonia e outro com 1516 crianças hospitalizadas por infecção pelo VSR, demonstrou-se que, além dos sinais de dispnéia, as alterações nas freqüências cardíaca e respiratória estiveram associadas a pior prognóstico ${ }^{19,20}$. Entretanto, a avaliação de 177 crianças holandesas hospitalizadas por 
infecção pelo VSR não encontrou maior risco de tempo prolongado de hospitalização para os critérios freqüência cardíaca e saturação menor de $92 \%$ à admissão 21 .

Como esperado, e em decorrência da maior gravidade dos pacientes internados, houve diferença significativa entre os dois grupos com relação ao tempo de hospitalização e de oxigenioterapia. O uso mais freqüente de antibioticoterapia no grupo de crianças submetidas à ventilação mecânica pode ser justificado pela apresentação mais grave da doença aguda pulmonar e pela dificuldade de estabelecer-se 0 diagnóstico de certeza da infecção bacteriana. Estes fatos podem ter contribuído para o uso empírico de antibióticos, com o objetivo de minimizar riscos. Os mesmos achados estão presentes em outros estudos, que avaliam estes aspectos evolutivos da doença aguda pulmonar $^{22,23,24}$. Em trabalho anterior que avaliou 684 crianças hospitalizadas com bronquiolite e pneumonia, há uma alta taxa de uso de antibioticoterapia, ao redor de 68\%, justificada por várias doenças intercorrentes, desde otite média aguda até sepse ${ }^{12}$.

O grupo de pacientes em VPMI também mostrou maior número de reinternações nos primeiros 30 dias. Estudos semelhantes mostraram evolução desfavorável em parte dos pacientes com doenças respiratórias baixas, com risco relativo maior para sibilância no primeiro ano de vida ${ }^{12,25,26,27}$ e para reinternações, no primeiro ano após a doença ${ }^{12}$. As possíveis lesões decorrentes da ventilação mecânica e do uso de oxigênio, o processo inflamatório pulmonar mais acentuado e a exposição contínua destes pacientes a estímulos virais, própria do primeiro ano de vida, podem explicar este achado.

\section{ConClusão}

Deste modo, do presente estudo conclui-se que, nas crianças previamente hígidas, com peso ao nascer e idade gestacional adequados, e com apresentação clínica de doença respiratória aguda baixa, deve-se focar a atenção nos lactentes muito jovens, com aleitamento curto ou ausente e na presença de cianose, como fatores ligados a uma evolução potencialmente desfavorável. Estudos com maior número de pacientes podem identificar ou ressaltar outros fatores prognósticos das infecções respiratórias agudas e traçar um melhor panorama da evolução a curto e médio prazo.

\section{Conflito de interesse: não há}

\section{SUMMARY}

\section{Prognostic factors for mechanical Ventilation in INFANTS WITH ACUTE LOWER RESPIRATORY DISEASE}

OBJECTNE. Acute lower respiratory tract infections are the most common cause of hospital admission in pediatrics. A number of admitted patients need invasive mechanical pulmonary ventilation (IMPV). This study aimed to evaluate prognostic factors for IMPV in infants admitted due to acute lower respiratory infection.

METHODS. A prospective cohort study was conducted from April to September, 2004, in two university hospitals of the Campinas metropolitan area, São Paulo, Brazil. One hundred, fifty-two infants were enrolled.
Epidemiological and clinical data were recorded at admission and follow-up. Two groups were analyzed, according to the need ofIMPV, with a comparison of prognostic factors. Association between risk factors and the outcome were studied and assessed by Relative Risk (RR), with confidence intervals of $(95 \% \mathrm{Cl})$.

RESULTS. Twenty-one patients (13.81\%) needed IMPV. Factors significantly associated with IMPV on admission were: age $<3$ months $(R R=2.35,95 \% C l: 1.06-5.22)$, breast feeding $<$ I month $(R R=3.15$, 95\% Cl:1.35-7.35) and cyanosis ( $R R=7.55,95 \% \mathrm{Cl}: 5.01-11.36)$. In the IMPV group, increased risks for hospitalization $>10$ days $(R R=13.69$, 95\% Cl:4.92-38.09), oxygen therapy $>10$ days $(R R=13.57$, 95\%Cl:5.4I-34.03), antibiotic usage $(R R=3.03,95 \%$ Cl: I.34-6.89) and readmission $(R R=5.23,95 \% \mathrm{Cl}: 2.12-12.91)$ were observed.

CONCLUSION. The associations between need of IMPV and early age, reduced breast feeding and cyanosis demonstrate diminished physiological reserves in the young infant with lower respiratory infection. These patients require prolonged and intensive hospital support and readmission. [Rev Assoc Med Bras 2006; 52(5): 342-6]

KEY WORDS: Acute respiratory infections. Mechanical ventilation. Pediatric intensive care. Children.

\section{REFERÊNCIAS}

I. Denny FW Jr. The impact of respiratory virus infections on the world's children. In: Skoner DP, editor. Asthma and respiratory infections. New York: Marcel Deker; 200 I. p. I-22.

2. Antuñano FJL. Epidemiologia de lãs infecciones respiratórias agudas em niños: panorama regional. In: Benguigui Y, Antuaño FJL, Schmunis G, Yunes J, editores. Infecciones respiratórias em niños. New York: Organización Panamericana de la Salud; 1997. p. 3-23.

3. WHO. World Health Organization. Initiative for Vaccine Research (IVR). Acute respiratory infections [cited 2004 aug 23]. Avaliable from: http:// www.who.int/vaccine_research/diseases/ari/en.

4. Denny FW. Acute respiratory infections in children: etiology and epidemiology (review). Pediatr Rev 1987;9: I35-46.

5. Júven T, Mertsola J, Waris M, Leinonen M, Meurman $\bigcirc$, Roivanen $M$, et al. Etiology of community-acquired pneumonia in 254 hospitalized children. Pediatr Infect Dis J 2000; 19:293-8.

6. Meissner HC. Uncertainty in the management of viral lower respiratory tract disease. Pediatr 2001; 108: 1000-4.

7. Wennergren G, Kristjansson S. Wheezing in infancy and its long-term consequences. Eur Resp Mon 2002; 19: I 16-30.

8. Gold DR, Burge HÁ, Carey V, Milton DK, Platts-Milss T, Weiss ST. Predictors of repeated wheeze in the first year of life. Am J Resp Crit Care Med 1999; 160:227-36.

9. Millán, T; Serani F; Vargas NA; Valenzuela MS. Características biológicas y sociales de los menores de un año muertos por neumonía en la región metropolitana de Chile, 1995. Rev Panam Salud Públ 1999;6:333-41.

10. Post CLA; Victora CG; Valente JG; Leal MC; Niobey, FML; Sabroza PC. Fatores prognósticos de letalidade hospitalar por diarréia ou pneumonia em menores de um ano de idade. Estudo de caso e controle. Rev Saúde Pública 1992;26:369-78.

I I. Sehgal V, Sethi GR, Sachved HPS, Satyanarayana L. Predictors of mortality in subjects hospitalized with acute lower respiratory tract infections. Indian Pediatr 1997;34:213-9.

12. Willson DF, Landrigan CP, Horn SD, Smout RJ. Complications in infants hospitalized for bronchiolitis or respiratory syncytial virus pneumonia. J Pediatr 2003; | 43:SI42-SI49. 
13. SEADE. Fundação Sistema Estadual de Análise de Dados. População/ Estatísticas vitais/SP Demográfico/Estatísticas Vitais do Estado de São Paulo. [citado 2004]. Disponível em: http://www.seade.gov.br.

14. Jorden RC: Typical vital signs in the pediatric population. In: Rosen P, Barkin $\mathrm{R}$, et al., editors. Multiple trauma in emergency medicine: concepts and clinical practice. $3^{\text {rd }}$ ed. St Louis: Mosby-Year Book, Inc; 1982. p.28I-2.

15. Rosenthal M, Bush A. The growing lung: normal development, and the long-term effects of pre and postnatal insults. Eur Respir Mon 2002;19:1-24

16. Pettigrew MM, Klodaee M, Gillespie B, Schwartz K, Bobo JK, Foxman B. Duration of breastfeeding, daycare and physician visits among infants 6 months and younger. Ann Epidemiol 2003; 13:43 I-5.

17. Albernazi EP, Menezes ANG, César JÁ, Victora CG, Barros FC, Helpern R. Risk factors associated with hospitalization for bronchiolitis in the postneonatal period. Rev Saúde Pública 2003;37:485-93.

18. Duarte, D.M.G. Perfil clínico de crianças menores de cinco anos com infecção respiratória aguda. J Pediatr(Rio de J) 2000;76:207- 12.

19. Wang EEL, Law BJ, Stephens D, Langley JM, MacDonald NE, Robinson JL, et al. Study of interobserver reliability in clinical assesment of RSV lower respiratory illness: a pediatric investigators collaborative network for infections in Canada (PICNIC) study. Pediatr Pulmonol 1996;22:23-7.

20. Shann, F.; Fracp, J.B.; Poore, P. Clinical signs that predict death in children with severe pneumonia. Pediatr Infect Dis J 1999:8:852-5.

2I. Kneyber MCJ, Moons KGM, De Groot R, Moll HA. Prediction of duration of hospitalization in respiratory syncytial virus infection. Pediatr Pulmonol 2002;33:453-7.
22. Farias JA, Frutos F, Esteban A, Casado Flores J, Retta A, Baltodano A, et al. What is the daily practice of mechanical ventilation in pediatric intensive care units? A multicenter study. Intensive Care Med 2004,30:9 I 8-25.

23. Roe M, O'Donnell DR, Tasker RC. Respiratory viruses in the intensive care unit. Paediat Respir Rev 2003,4: I66-7|

24. Panitch HB. Respiratory syncytial virus bronchiolitis: supportive care and therapies designed to overcome airway obstrution. Pediatr Infect Dis J 2003;22:S83-8.

25. Sigurs N, Bjarnason R, Sigurbergsson F, Kjellman B, Björkstén B. Asthma and immunoglobulin $E$ antibodies after respiratory syncytial virus bronchiolitis: a prospective cohort study with matched controls. Pediatrics 1995;95:500-5.

26. Martinez FD, WrightAll, Taussig LM, Holberg CJ, Halonen M, Morgan WJ. Asthma and wheezing in the first six years of life. $N$ Engl J Med 1995;332:133-8

27. Stein RT, Sherrill D, Morgan WJ, Holberg CJ, Halonen M, Taussig LM, et al. Respiratory syncytial virus in early life and risk of wheeze and allergy by age I3 years. Lancet 1999; 1 54:54 I-5.

Artigo recebido: 27/06/2005

Aceito para publicação: 28/12/2005 